\title{
A wake-up call: persistent barriers to the provision of evidence- based lactation support and education in the NICU
}

\author{
Beverly Rossman ${ }^{1} \cdot$ Paula P. Meier ${ }^{2} \cdot$ Diane L. Spatz $^{3}$
}

Received: 23 February 2018 / Accepted: 20 March 2018 / Published online: 22 June 2018

(c) Nature America, Inc., part of Springer Nature 2018

Mothers who give birth to infants cared for in the neonatal intensive care unit (NICU) experience barriers to the achievement of their personal breastfeeding goals that are not shared by mothers of term, healthy infants. Specifically, these mothers are breast pump dependent, must cope simultaneously with the stress of the NICU hospitalization and the infant's uncertain condition, and are frequently sick themselves. Even with evidence-based, NICU-specific lactation care, these mothers do not maintain lactation at rates that are comparable to healthy populations [1-3]. The primary reason for lack of achievement of individual breastfeeding goals is inadequate mother's own milk (MOM) volume to meet their infants' nutritional needs [4]. The extent to which inadequate MOM volume over the NICU hospitalization is due to maternal risk factors such as obesity and cesarean delivery, the profound dislike of breast pump use, the inability to use the pump frequently enough to regulate lactation processes, the stress and/or discouragement with low pumped MOM volumes, or lack of evidence-based information and support from NICU care providers is unknown.

Last month, Demirci et al. [5] reported a subgroup of NICU mothers-those who give birth to infants with complex surgical anomalies-and have chosen a qualitative methodology to report these 15 mothers' perceptions and experiences with respect to providing MOM during the first 2 months post birth. In this 55-bed, Level IV NICU,

Beverly Rossman

brossman@comcast.net

Beverly_Rossman@rush.edu

1 Department of Women, Children and Family Nursing, Rush University Medical Center, 600S Paulina, Suite 1080, Chicago, IL 60612, USA

2 Department of Pediatrics, Rush University Children's Hospital, 1653 West Congress Parkway, Chicago, IL 60612, USA

3 Children's Hospital of Philadelphia, 418 Curie Blvd, Room 413, Philadelphia, PA 19104, USA mothers participated in one to three in-depth interviews during their infants' NICU hospitalization and transition to home. Although the NICU had recently instituted a quality improvement initiative targeting improvement in MOM provision rates, the study reported that the mothers had a fundamental lack of understanding about the importance of MOM for their surgical infants. Most maternal perceptions and experiences were consequent to this lack of effective messaging. Among the mothers' general desire for more help and information with all aspects of providing MOM, the primary specific concerns focused on why exclusive MOM feedings were necessary, MOM volume, assistance with the basics of direct breastfeeding, and emotional support and encouragement for coping with the lifestyle challenges inherent in breast pump dependency.

The Demirici et al. [5] findings also highlight the difficulties in translating published research, such as the "Transition to Breast Pathway" [6], into practice. Few of the mothers in the Demirci et al. [5] study engaged in skin-toskin care, oropharyngeal colostrum administration, and nonnutritive sucking, strategies known to increase the probability that mothers of surgical infants meet their personal breastfeeding goals and directly breastfeed their infants prior to discharge [6].

Furthermore, the early post-birth period, which coincides with the early phases of lactation of initiation and coming to volume, is fraught with difficulties for all mothers, but is especially problematic for mothers of NICU infants because they often receive inappropriate advice and equipment (e.g., non-hospital-grade electric breast pumps), compromising long-term MOM volume and exclusive breastfeeding [7, 8]. In the absence of proactive evidence-based lactation care by NICU staff, it is likely that mothers' dislike of and frustration with the lifestyle inconveniences with pumping play a role in decreased milk volumes and decisions to discontinue providing MOM.

The Demirici et al. [5] results should serve as a wake-up call for all healthcare providers who work with mothers of surgical infants cared for in the NICU. Basic strategies to 
protect maternal milk volume and prevent or detect common lactation problems within the first 14 days post birth for breast pump-dependent mothers in the NICU have been known for more than a decade. Two models of effective lactation care for NICU mothers have been implemented and evaluated in the research literature: The Rush Mothers' Milk Club which incorporates breastfeeding peer counselors (former NICU parents themselves) as primary lactation care providers [7, 9, 10]; and the Breastfeeding Resource Model (BRN), which expands the role of the bedside nurse as expert lactation care provider by following "10 steps for promoting and protecting breastfeeding in vulnerable infants" and a "Transition to Breast Pathway" for mothers of surgical infants $[6,11,12]$. Implementation of the BRN and Spatz 10-step model has resulted in consistently high human milk rates at discharge $(83-86 \%)$ [13]. Common to both models is the provision of accurate, comprehensive, evidence-based lactation care and support occurring within an established institutional culture of using the evidence about human milk in the NICU and disseminating this information to mothers and families [9]. Mothers have an expectation that their lactation support needs will be met while their infant is hospitalized. The motivation to meet this expectation and ensure that every mother understands the importance of human milk for their infant should become a priority for all NICU quality improvement initiatives.

\section{Compliance with ethical standards}

Conflict of interest The authors declare that they have no conflict of interest.

\section{References}

1. Davanzo R, Monasta L, Ronfani L, Brovedani P, Demarini S, Breastfeeding in Neonatal Intensive Care Unit Study Group.
Breastfeeding at NICU discharge: a multicenter Italian study. J Hum Lact. 2013;29:374-80.

2. Pineda RG. Predictors of breastfeeding and breastmilk feeding among very low birth weight infants. Breastfeed Med. 2011;6: 15-19.

3. Perrine CG, Scanlon KS. Prevalence of use of human milk in US advanced care neonatal units. Pediatrics. 2013;131: 1066-71.

4. Hoban R, Bigger H, Schoeny M, Engstrom J, Meier P, Patel AL. Milk volume at 2 weeks predicts mother's own milk feeding at neonatal intensive care unit discharge for very low birthweight infants. Breastfeed Med. 2018;13:135-41.

5. Demirci J, Caplan E, Brozanski B, Bogen D. Winging it: maternal perspectives and experiences of breastfeeding newborns with complex surgical anomalies. J. Perinatol. 2018;38: 708-17.

6. Edwards TE, Spatz DL. An innovative model for achieving breastfeeding success in infants with complex surgical anomalies. J Perinat Neonatal Nurs. 2010;24:254-5.

7. Meier PP, Patel AL, Bigger HR, Rossman B, Engstrom JL. Supporting breastfeeding in the neonatal intensive care unit: Rush Mother's Milk Club as a case study of evidence-based care. Pediatr Clin North Am. 2013;60:209-26.

8. Meier PP, Patel AL, Hoban R, Engstrom JL. Which breast pump for which mother? An evidence based approach to individualizing breast pump technology. J Perinatol. 2016;36:493-9.

9. Meier PP, Engstrom JL, Rossman B. Breastfeeding peer counselors as direct lactation care providers in the neonatal intensive care unit. J Hum Lact. 2013;29:313-22.

10. Rossman B, Engstrom JL, Meier PP, Vonderheid SC, Norr KF, Hill PD. "They've walked in my shoes": mothers of very low birth weight infants and their experiences with breastfeeding peer counselors in the neonatal intensive care unit. J Hum Lact. 2011;27:14-24.

11. Spatz DL. Ten steps for promoting and protecting breastfeeding for vulnerable infants. J Perinat Neonatal Nurs. 2004;18: 385-96.

12. Spatz DL, Froh EB, Flynn-Roth R, Barton S. Improving practice at the point of care through the optimization of the breastfeeding resource nurse model. J Obstet Gynecol Neonatal Nurs. 2015;44:412-8.

13. Spatz, DL. Beyond BFHI: The Spatz 10 step \& breastfeeding resource nurse model to improve human milk and breastfeeding outcomes. J Perinat Neonatal Nurs. 2018; in press. 